\title{
Functional Testing of Digital Microfluidic Biochips*
}

\author{
Tao Xu and Krishnendu Chakrabarty \\ Department of Electrical and Computer Engineering \\ Duke University, Durham, NC 27708, USA \\ $\{$ tx, krish\}@ee.duke.edu
}

\begin{abstract}
Dependability is an important attribute for microfluidic biochips that are used for safety-critical applications such as point-of-care health assessment, air-quality monitoring, and food-safety testing. Therefore, these devices must be adequately tested after manufacture and during bioassay operations. Known techniques for biochip testing are all function-oblivious, i.e., while they can detect and locate defect sites on a microfluidic array, they cannot be used to ensure correct operation of functional units. In this paper, we introduce the concept of functional testing of microfluidic biochips. We address fundamental biochip operations such as droplet dispensing, droplet transportation, mixing, splitting, and capacitive sensing. Long electrode actuation times are avoided to ensure that there is no electrode degradation during testing. We evaluate the proposed test methods using simulations as well as experiments for a fabricated biochip.
\end{abstract}

\section{Introduction}

Microfluidics-based biochips, also referred to as lab-on-a-chip, are replacing cumbersome and expensive laboratory equipment for applications such as high-throughput sequencing, parallel immunoassays, protein crystallization, blood chemistry for clinical diagnostics, and environmental toxicity monitoring $[1,2,3]$. These miniaturized and automated biochip devices offer the advantages of higher sensitivity, lower cost due to smaller sample and reagent volumes, higher levels of system integration, and less likelihood of human error.

A popular class of microfluidic biochips is based on continuous fluid flow in permanently-etched microchannels. These devices rely on either micropumps and microvalves to electrical methods based on electrokinetics to control continuous sample flows [2]. Specific to electroosmosis flows, a MOSFET-like design, referred to as a FlowFET, has also been proposed [4].

An alternative category of microfluidic biochips relies on "digital microfluidics", which is based on the principle of electrowetting-on-dielectric [1]. By manipulating discrete droplets of nanoliter volume using a patterned array of electrodes, miniaturized bioassay protocols (in terms of liquid volumes and assay times) are mapped and

*This work was supported in part by the National Science Foundation under grants IIS-0312352 and CCF-0541055. executed on a microfluidic chip. Therefore, digital microfluidic biochips require only nanoliter volumes of samples and reagents. They offer continuous sampling and analysis capabilities for on-line and real-time chemical/biological sensing. These systems also have a desirable property referred to as dynamic reconfigurability, whereby microfluidic modules can be relocated to other places on the electrode array, without affecting functionality, during the concurrent execution of a set of bioassays [4]. Reconfigurability enables microfluidic biochips to be "adaptive" to a wide variety of applications. System reconfiguration can also be used to bypass the faulty cells to enable microfluidic arrays to provide reliable results even in the presence of defects.

Recent years have seen rapid progress in the mapping of different bioassays for concurrent execution on digital microfluidic biochips $[5,6]$. As a result, system complexity and integration levels are increasing steadily. As in the case of integrated circuits (ICs), an increase in the density and area of microfluidics-based biochips will lead to high defect densities, thereby reducing yield, especially for newer technologies. However, dependability is an important system attribute for biochips. Dependability is essential for safety-critical applications such as point-of care diagnostics, health assessment and screening for infectious diseases, air-quality monitoring, and food-safety tests, as well as for pharmacological procedures for drug design and discovery that require high precision levels. Some manufacturing defects may be latent, and they may produce errors during field operation. In addition, harsh operational environments and biological samples (e.g., proteins) may introduce physical defects such as particle contamination and residue on surfaces due to adsorption.

Since complicated fluidic operations are repeatedly executed with high precision in compact microfluidic arrays, a group of cells is repeatedly required to perform a large number of operations. Traditional structural test methods, which use test droplets to traverse the target array, do not provide a sufficient level of confidence that these fluidic operations can be reliably performed on the array [7]. For instance, some unit cells, i.e., electrodes, may function correctly during droplet transportation, but they might malfunction during droplet dispensing from reservoirs. Likewise, unit cells that can be reliably combined to operate as a mixer may malfunction when they are used for droplet splitting. Moreover, a structural test does not cover non-reconfigurable modules such as capacitive sensing circuits. A defect involving any of the modules may result 
in catastrophic failure during bioassay execution. Therefore, before we use synthesis methods to map bioassay protocols to a microfluidic array [8], it is important to carry out functional testing to verify the integrity of the underlying microfluidic platform. To ensure that manufactured biochips are competitive in the emerging low-cost market for disposable biochips and to avoid electrode degradation resulting from excessive actuation, test methodologies should be inexpensive, quick, and effective.

In this paper, we first present various defects that are typical for digital microfluidic biochips. We relate these defects to logical fault models that can be viewed not only in terms of traditional shorts and opens, but which also target biochip functionality. Based on these fault models, we introduce the idea of functional testing of digital microfluidic modules. We develop cost-effective functional test methods that target fluidic operations such as droplet dispensing, droplet transportation, mixing, and splitting. These methods also test the functionality of non-reconfigurable modules such as capacitive sensing circuits. The proposed methods allow functional testing using parallel droplet pathways in both on-line and off-line scenarios. For each function, the proposed approach identifies "qualified regions", i.e., groups of cells that pass the test. Instead of placing fluidic modules in a fault-oblivious manner on the microfluidic array, synthesis tools can map modules only to qualified regions. In this way, the reliability of the synthesized biochip is significantly increased. We evaluate the proposed functional test methods using simulations as well as experiments for a fabricated biochip.

The rest of the paper is organized as follows. Section 2 provides an overview of digital microfluidic biochips. In Section 3, we discuss related prior work on defect modeling and structural testing. In Section 4, we relate defects to fault models and observable errors, and we list various fault models for digital microfluidic biochips. We next introduce the concept of functional testing and propose effective methods to test the biochip for droplet dispensing, droplet transportation, mixing, splitting, and capacitive sensing. In Section 5, we apply these techniques to a fabricated chip and present simulation results. Finally, conclusions are drawn in Section 6.

\section{Digital Microfluidic Biochips}

A digital microfluidic biochip utilizes the phenomenon of electrowetting to manipulate and move nanoliter droplets containing biological samples on a two-dimensional electrode array [1]. A unit cell in the array includes a pair of electrodes that acts as two parallel plates. The bottom plate contains a patterned array of individually controlled electrodes, and the top plate is coated with a continuous ground electrode. A droplet rests on a hydrophobic surface over an electrode, as shown in Fig. 1. It is moved by applying a control voltage to an electrode adjacent to the droplet and, at the same time, deactivating the electrode just under the droplet. This electronic method of wettability control creates interfacial tension gradients that move the

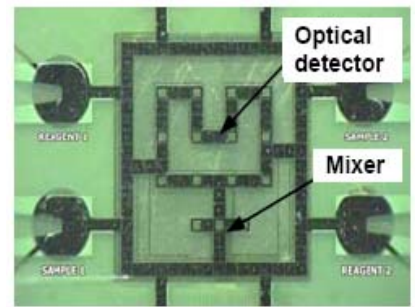

Glass-substrate platform

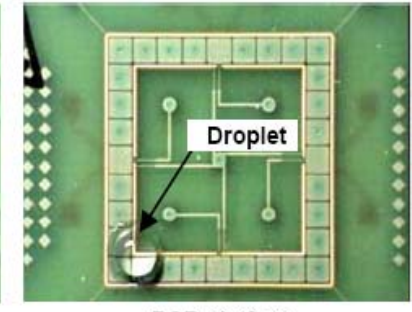

PCB platform
Fig. 1: Fabricated digital microfluidic arrays.

droplets to the charged electrode. Using the electrowetting phenomenon, droplets can be moved to any location on a two-dimensional array.

By varying the patterns of control voltage activation, many fluid-handling operations such as droplet merging, splitting, mixing, and dispensing can be easily executed. For example, mixing can be performed by routing two droplets to the same location and then turning them about some pivot points. The digital microfluidic platform offers the additional advantage of flexibility, referred to as dynamic reconfigurability, since fluidic operations can be performed anywhere on the array. Droplet routes and operation scheduling result are programmed into a microcontroller that drives electrodes in the array. In addition to electrodes, optical detectors such as LEDs and photodiodes are also integrated in digital microfluidic arrays to monitor colorimetric bioassays [3].

\section{Related Prior Work}

The testing of microfluidic biochips has recently been investigated. These test methods add fluid handling aspects to MEMS testing techniques $[9,10]$. Test methods have been proposed for both continuous-flow and digital microfluidic biochips. An excellent review is available in [11]. A fault model and a fault simulation method for continuous-flow microfluidic biochips have been proposed in [12]. For digital microfluidic chips, techniques for defect classification, test planning, and test resource optimization have been presented [7]. Defect classification methods are discussed in [7] and corresponding test procedures are described in [13]. Defects have been classified as being either catastrophic or parametric, and techniques have been developed to detect these defects by electrostatically controlling and tracking droplet motion.

The work in $[7,13]$ facilitates concurrent testing, which allows fault detection and biomedical assays to run simultaneously on a microfluidic system [14]. A drawback of [7] however is that it does not present any automated techniques for optimizing the test application procedure. [15] first proposed a test planning and test resource optimization method. The test planning problem is mapped to the Hamilton cycle problem from graph theory. An alternative method based on Euler paths is proposed in [16]. This method maps a digital microfluidic biochip to an undirected graph and a test droplet is routed along the Euler path derived from the graph to pass through all the cells in the array. Fault diagnosis is carried out using multiple test application steps and adaptive Euler paths.

A drawback of the above "structural" test methods is 
Table 1: Fault models for digital microfluidic biochips.

\begin{tabular}{|l|l|c|l|l|}
\hline $\begin{array}{l}\text { Cause of } \\
\text { defect }\end{array}$ & $\begin{array}{l}\text { Defect } \\
\text { type }\end{array}$ & $\begin{array}{l}\text { Number } \\
\text { of } \text { cells }\end{array}$ & $\begin{array}{l}\text { Fault } \\
\text { model }\end{array}$ & $\begin{array}{l}\text { Observable } \\
\text { error }\end{array}$ \\
\hline $\begin{array}{l}\text { Excessive actuation } \\
\text { voltage applied to an electrode }\end{array}$ & Dielectric breakdown & 1 & $\begin{array}{l}\text { Droplet-electrode short (a } \\
\text { short between the droplet } \\
\text { and the electrode) }\end{array}$ & $\begin{array}{l}\text { Droplet undergoes electrolysis, which } \\
\text { prevents its further transportation }\end{array}$ \\
\hline $\begin{array}{l}\text { Electrode actuation for } \\
\text { excessive duration }\end{array}$ & $\begin{array}{l}\text { Irreversible charge } \\
\text { concentration on an electrode }\end{array}$ & 1 & $\begin{array}{l}\text { Electrode-stuck-on (the } \\
\text { electrode remains constantly } \\
\text { activated) }\end{array}$ & $\begin{array}{l}\text { Unintentional droplet operations or } \\
\text { stuck droplets }\end{array}$ \\
\hline $\begin{array}{l}\text { Excessive mechanical force } \\
\text { applied to the chip }\end{array}$ & $\begin{array}{l}\text { Misalignment of parallel plates } \\
\text { (electrodes and ground plane) }\end{array}$ & 1 & $\begin{array}{l}\text { Pressure gradient (net static } \\
\text { pressure in some direction) }\end{array}$ & $\begin{array}{l}\text { Droplet transportation without } \\
\text { activation voltage }\end{array}$ \\
\hline $\begin{array}{l}\text { Coating failure } \\
\text { Non-uniform dielectric layer }\end{array}$ & 1 & $\begin{array}{l}\text { Dielectric islands } \\
\text { (islands of Teflon coating) }\end{array}$ & $\begin{array}{l}\text { Fragmentation of droplets and their } \\
\text { motion is prevented }\end{array}$ \\
\hline $\begin{array}{l}\text { Abnormal } \\
\text { metal layer } \\
\text { deposition } \\
\text { and etch } \\
\text { variation } \\
\text { during } \\
\text { fabrication }\end{array}$ & Grounding Failure & 1 & $\begin{array}{l}\text { Floating droplets (droplet are } \\
\text { not anchored })\end{array}$ & Failure of droplet transportation \\
\cline { 2 - 5 } & Broken wire to control source & 1 & $\begin{array}{l}\text { Electrode open (electrode } \\
\text { actuation is not possible) }\end{array}$ & $\begin{array}{l}\text { Failure to activate the } \\
\text { electrode for droplet } \\
\text { transportation }\end{array}$ \\
\hline $\begin{array}{l}\text { Particle contamination or } \\
\text { liquid residue }\end{array}$ & $\begin{array}{l}\text { Metal connection between two } \\
\text { adjacent electrodes }\end{array}$ & 2 & $\begin{array}{l}\text { Electrode short (short } \\
\text { between electrodes) }\end{array}$ & $\begin{array}{l}\text { A droplet resides in } \\
\text { the middle of the two shorted } \\
\text { electrodes, and its transport along one } \\
\text { ad more directions } \\
\text { cannot be achieved }\end{array}$ \\
\hline $\begin{array}{l}\text { Protein adsorption during } \\
\text { bioassay [17] }\end{array}$ & $\begin{array}{l}\text { Sample residue on electrode } \\
\text { surface }\end{array}$ & 1 & Resistive open at electrode & Droplet transportation is impeded. \\
\cline { 2 - 5 } & Contamination & $\begin{array}{l}\text { Assay results are outside the range of } \\
\text { possible outcomes }\end{array}$ \\
\hline
\end{tabular}

\begin{tabular}{|l|l|l|l|l|}
\hline $\begin{array}{l}\text { Cause of } \\
\text { malfunction }\end{array}$ & $\begin{array}{l}\text { Malfunction } \\
\text { type }\end{array}$ & $\begin{array}{l}\text { Number } \\
\text { of cells }\end{array}$ & $\begin{array}{l}\text { Fault } \\
\text { model }\end{array}$ & $\begin{array}{l}\text { Observable } \\
\text { error }\end{array}$ \\
\hline $\begin{array}{l}\text { Electrode actuation for } \\
\text { excessive duration }\end{array}$ & $\begin{array}{l}\text { Irreversible charge } \\
\text { concentration on the dispensing } \\
\text { electrode }\end{array}$ & 3 & $\begin{array}{l}\text { Dispensing-stuck-on (droplet } \\
\text { is dispensed by not fully cut } \\
\text { off from the reservoir) }\end{array}$ & $\begin{array}{l}\text { No droplet can be dispensed from the } \\
\text { reservoir }\end{array}$ \\
\hline $\begin{array}{l}\text { Electrode shape variation in } \\
\text { fabrication }\end{array}$ & Deformity of electrodes & 3 & $\begin{array}{l}\text { No overlap between droplets } \\
\text { to be mixed and center } \\
\text { electrode }\end{array}$ & Mixing failure \\
\hline $\begin{array}{l}\text { Electrode electrostatic } \\
\text { property variation in } \\
\text { fabrication }\end{array}$ & Unequal actuation voltages & 3 & $\begin{array}{l}\text { Pressure gradient (net static } \\
\text { pressure in some direction) }\end{array}$ & Unbalanced volumes of split droplets \\
\hline Bad soldering & $\begin{array}{l}\text { Parasitic capacitance in the } \\
\text { capacitive sensing circuit }\end{array}$ & 1 & $\begin{array}{l}\text { Oversensitive or insensitive } \\
\text { capacitive sensing }\end{array}$ & False positive/negative in detection \\
\hline
\end{tabular}

that they focus only on fault modeling, and the test and diagnosis of physical defects, and they overlook module functionality. Therefore, these methods can only guarantee that a biochip is defect-free. However, a defect-free microfluidic array can also malfunction in many ways. For example, a defect-free reservoir may result in large volume variations when droplets are dispensed from it. A splitter composed of three defect-free electrodes may split a big droplet into two droplets with significantly unbalanced volumes. These phenomena, referred to as malfunctions, are not the result of electrode defects. Instead, they are activated only for certain patterns of droplet movement or fluidic operations. Such malfunctions can have serious consequences on the integrity of bioassay results. Therefore, to ensure robust execution of the target bioassay, we must carry out more comprehensive test procedures, which not only target defective cells, but also lead to the detection of malfunctioning microfluidic modules.

\section{Functional Testing}

In this section, we propose a comprehensive test procedure, referred to as functional testing, which targets the functional operation of microfluidic modules. To avoid ambiguity, we refer to the test methods discussed in
$[7,13,14]$ as structural test, since they route test droplets to all the electrodes in the array to ensure structural integrity. Structural test targets at physical defects, which are defined as the underlying cause of erroneous chip operation, where the defect affects either a unit cell (electrode) or the electrical connection to the unit cell. Functional testing, on the other hand, involves test procedures to check whether groups of cells can be used to perform certain operations, e.g., droplet mixing and splitting. For the test of a specific operation, the corresponding patterns of droplet movement are carried out on the target cluster of cells. If a target cell cluster fails the test, e.g., the mixing test, we label it as a malfunctioning cluster, which implies that the synthesis tool cannot place the corresponding module - a mixer in this case-in this region.

As in the case of structural testing, we first develop a fault model for functional testing. Since functional testing is an extension of structural testing, all the defect-oriented fault models for also included here. Therefore, we start from the fault models proposed in [8]. Malfunctions in fluidic operations are then identified and added to the list. In this way, we derive a more comprehensive set of fault models, as shown in Table 1. 


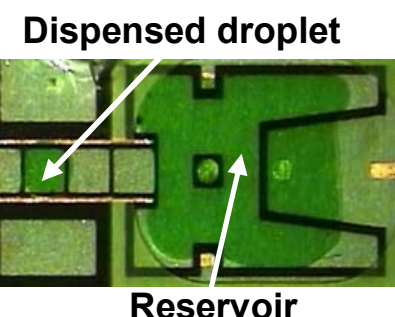

(a)

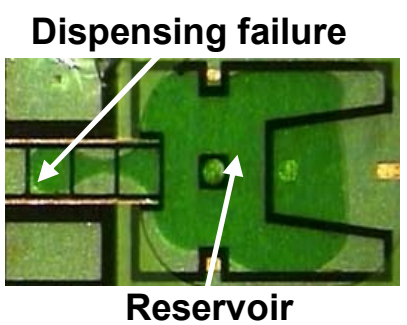

(b)

Fig. 2: Illustration of (a) normal dispensing and (b) dispensing failure, for a fabricated microfluidic biochip.

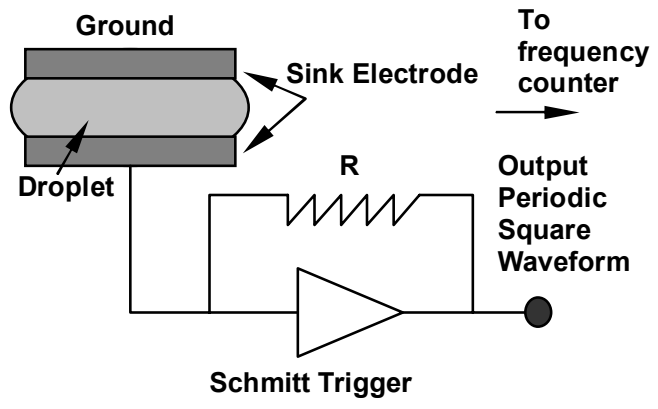

(a)

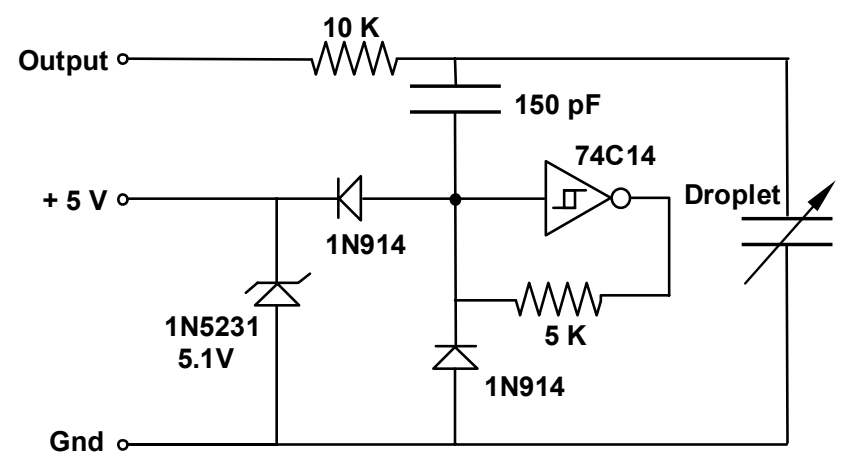

(b)

Fig. 3: Capacitive sensing circuit (a) outline [13] (b) detail circuit.

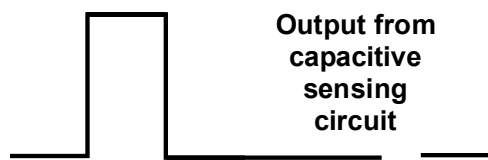

(a)

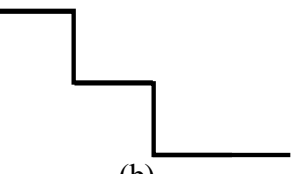

(b)
Fig. 4: Test readouts for (a) normal dispensing and (b) dispensing failure.

Next we propose efficient functional test methods to detect the defects and malfunctions listed in Table 1. Dispensing test, mixing test, splitting test, and capacitive sensing test are developed to address the corresponding malfunctions. A routing test procedure is used to detect all physical defects.

\subsection{Dispensing Test}

Dispensing test targets the malfunctioning of the dispensing operation. Fig. 2 provides a comparison between normal dispensing and an example of dispensing failure. As shown in Fig. 2(b), the dispensed droplet in a malfunctioning scenario cannot be detached from the droplet in the reservoir. Therefore, when we move the dispensed droplet away from the reservoir, an additional droplet from the reservoir is extracted and moved as well. In this case, the dispensed "droplet" can be several times larger than the normal size, which may result in the catastrophic failure of a volume-sensitive bioassay.

Here we propose a test method based on capacitive sensing to detect these dispensing failures. The circuit diagram for fault detection is shown in Fig. 3. It has been shown in the literature that dispensing involves a reservoir and the three electrodes closest to it [18]. We therefore define every reservoir together with its three neighbor electrodes as a dispensing cluster. The third electrode in the cluster, i.e., the electrode farthest from the reservoir, is connected to a capacitive sensing circuit for test readout. When the test starts, a droplet is dispensed from the reservoir until it reaches the third electrode. According to Fig. 2, we expect a positive pulse with normal amplitude for both fault-free dispensing and dispensing failure. Next, we route the dispensed droplet one electrode in the forward direction. During correct dispensing, the fully-dispensed droplet moves completely to the fourth electrode, thereby no pulse is detected by the capacitive sensing circuit output at this time. However, if the droplet undergoes a dispensing failure and it is still connected to the liquid in the reservoir, there must be some fluid left at the third electrode, which is indicated by a positive pulse, with smaller amplitude, in the test readout. Therefore, we can easily detect a dispensing failure by reading the output of the capacitive sensing circuit, as shown in Fig. 4.

To identify abnormal droplets, two threshold values for the pulse amplitude are used. These thresholds are determined through calibration of the sensing circuit. First, we fix a nominal value $\mu$ and a maximum allowable droplet volume variance $\sigma$. Then two droplets with volume of $\mu+\sigma$ and $\mu-\sigma$ are routed to the sensing circuit. Signal levels are recorded and used as the upper and lower threshold values respectively.

\subsection{Routing Test and Capacitive Sensing Test}

Routing test focuses on evaluating a single electrode's ability to transport droplets. This procedure is similar to that proposed earlier for structural test [7,13,14]. In structural test, a test droplet is dispensed and routed to cross the target electrode from two orthogonal directions, i.e., along the row and the column directions. The routing problem can be solved by mapping the array to an undirected graph and applying the Euler-path based method [11], as shown in Fig. 5. On the other hand, a test droplet must be routed along all four directions relative to the target electrode. We can solve the route planning problem in this case by mapping the target array to a directed graph, which can be easily derived by replacing every edge in the undirected graph with two directed edges in opposite directions. The Euler-path based method is then applied to the directed graph to derive a test plan for routing test.

Note that in structural test, a test droplet is routed to 

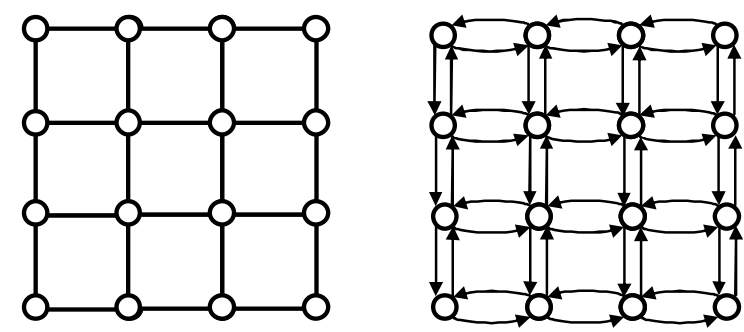

Fig. 5: (a) An undirected graph for Euler-path-based structural test; (b) The corresponding directed graph for Euler-path-based routing test.

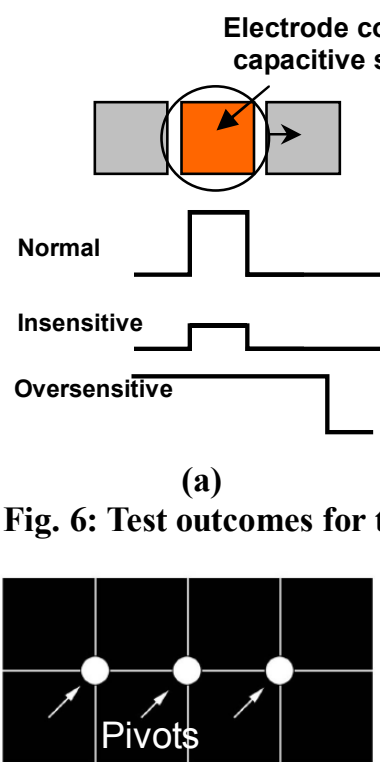

(a)

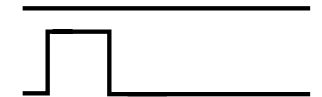

(b)

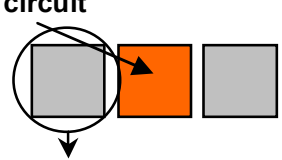

output

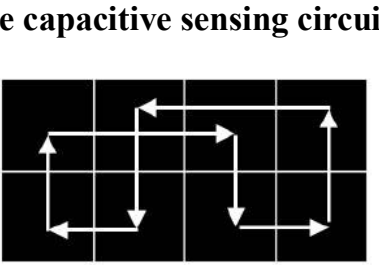

(b)

Fig. 7: (a) Pivots and (b) routing path for a $2 \times 4$ microfluidic mixer.

traverse the array, following the Euler path derived from the undirected graph in Fig. 5(a). As a result of this procedure, half of the directed edges in Fig. 5(b) are also traversed, with exactly one edge visited for each pair of directed edges between two nodes. The edges that are not visited can be ordered to form a "reverse path" corresponding to the Euler path derived above. Therefore, routing test can be carried out by applying two iterations of structural test in opposite directions. Recall that all the defects listed in Table 1 can be detected by structural test; therefore, they are also detected by the above routing test procedure.

The above test procedure also tests the functionality of the capacitive sensing circuit. If a test droplet is routed to visit the electrode connected to the target capacitive sensing circuit, a positive pulse is expected at the output of the sensing circuit. By examining the amplitude of the positive pulse, we can determine whether a capacitive sensing circuit is normal, insensitive, or oversensitive, as shown in Fig. 6.

\subsection{Mixing and Splitting Test}

Next we discuss the functional testing of two widely

used microfluidic modules - mixers and splitters. In a digital microfluidic biochip, two droplets are mixed within a cluster of electrodes, referred to as mixer. Even though mixer designs and configurations vary considerably $[19,20]$, the underlining mixing mechanisms remain the same for all designs and configurations. Two droplets are merged at one electrode and routed to move about some pivots in the mixer, as shown in Fig. 7.

Thus a mixing functional test is equivalent to the testing of the merging and routing operations within the target cell cluster. Recall that droplet routing test has been addressed in Section 4.2; therefore, a mixing test can be reduced to a droplet merging test, which checks a series of three adjacent electrodes to determine whether two droplets can be merged on them. For a microfluidic array, a simple test method carries out droplet merging on every group of three adjacent electrodes, one at a time. For such a three-electrode test, the test outcome is read out using a capacitive sensing circuit connected to the center electrode, on which droplets are supposed to be merged, as shown in Fig. 8. However, since every electrode can be the center of a set of three-electrodes, we have to connect a capacitive sensing circuit to it, which results in high production cost. Moreover, the serial processing method requires a large number of droplet manipulation steps and electrode actuations. As shown in Table. 1, excessive actuation may result in a variety of catastrophic defects. Therefore, efficient algorithms are needed for droplet-merging test.

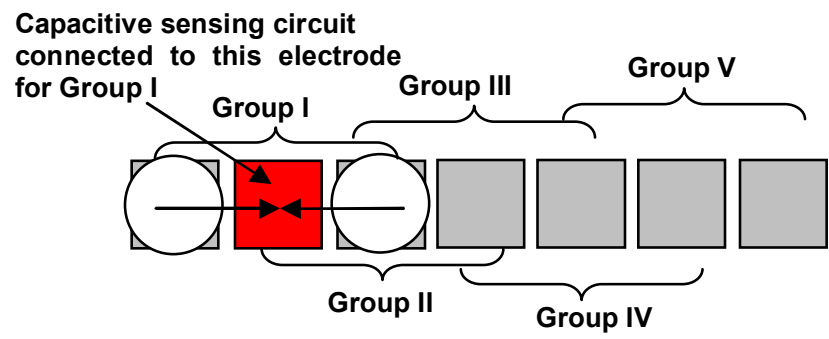

Fig. 8: Example of merging test.

Droplet splitting is simpler compared to mixing. The fluidic operation involves three adjacent electrodes. By applying an appropriate electrode-actuation sequence, a droplet that rests on the center electrode is split into two smaller droplets, which rest on the two side electrodes. Thus a split operation can be viewed as reverse of droplet merging. Consequently, splitting test can be carried out by applying the merging test methods in a reverse manner. The only difference lies in the fact that instead of connecting a capacitive sensing circuit to the center electrode, splitting test attaches two capacitive sensing circuits to the two side electrodes. The test outcome is evaluated by comparing output amplitudes of the two sensing circuits.

We next combine these two tests into a unified test application procedure. We start from the simple case where mixing and splitting test are carried out for two three-electrode groups centered at one electrode. For simplicity we limit our discussion to linear merging and splitting, i.e., the electrodes involved are linearly aligned in 


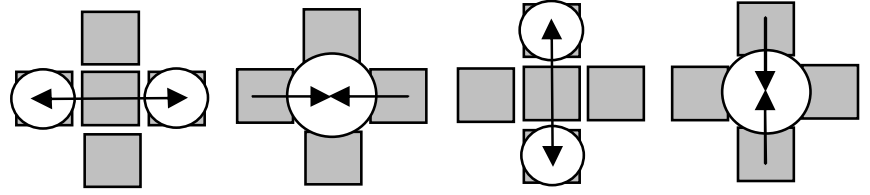

Fig. 9: Mixing and splitting test for all the groups of three electrodes that are centered on a given electrode.

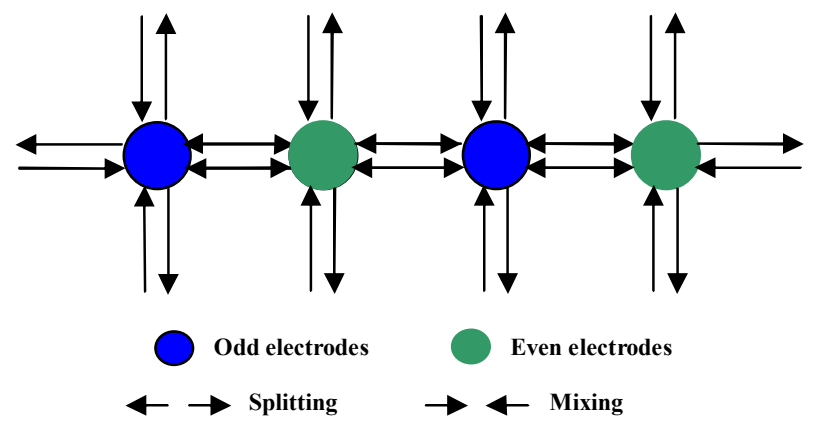

Fig. 10: Parallel mixing and splitting test for a row of electrodes.

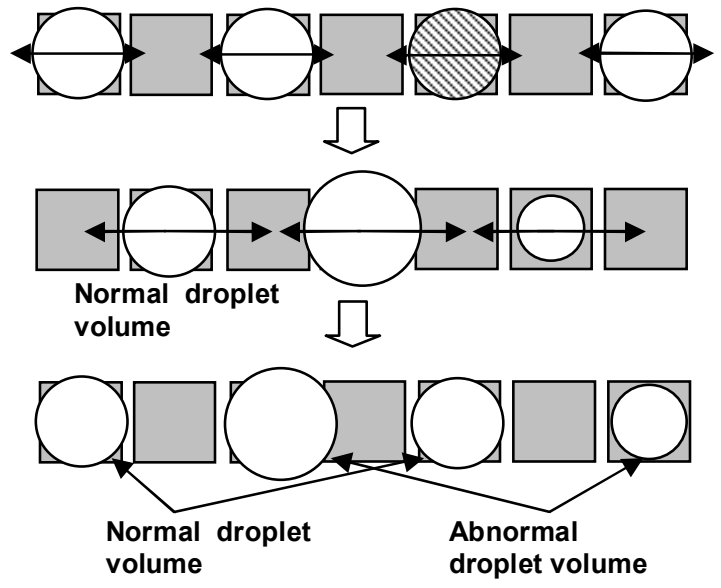

Fig. 11: Parallel mixing and splitting test for a row of electrodes.

the same row/column. The test procedure is illustrated in Fig. 9.

In Fig. 9, we carry out mixing and splitting test using four steps, i.e., horizontal splitting, horizontal mixing, vertical splitting and vertical mixing. Note that the ordering is carefully chosen such that the four steps can be carried out consecutively, with no additional routing steps needed in between. However, this procedure still requires every electrode to be connected to a capacitive sensing circuit. Moreover, in order to extend this test scheme to a microfluidic array, we need $4 N^{2}$ manipulation steps for an $N \times N$ array of electrodes, which is very inefficient.

To achieve higher test efficiency and lower hardware cost, we apply the single-electrode test methods in parallel for array testing. The key idea is to carry out mixing and splitting test for all the electrodes in a row/column concurrently. For simplicity of analysis, we demonstrate the method using a directed graph, where each electrode is mapped to a node in the graph, and each mixing or splitting test step is represented by a pair of directed edges; see Fig. 10.

The electrodes in Fig. 10 are labeled as being either "even" or "odd". We carry out the horizontal splitting test for all the even electrodes concurrently. The split droplets get merged at the odd electrodes, therefore the merging test is done at the same time. Similarly, by carrying out the splitting test for all the odd electrodes concurrently, we can easily complete the horizontal merging test for all the even electrodes. Thus we can carry out all the horizontal tests (merging and splitting) in one row using only two manipulation steps. Similarly, all the vertical tests in one column can be completed in two manipulation steps.

Following the above observations, we propose a parallel procedure to carry out mixing and splitting tests efficiently. The steps of the procedure are as follows:

1. Route large droplets to all the even electrodes in a row.

2. Carry out splitting test for all the even electrodes concurrently (large droplets are now on odd electrodes).

3. Carry out splitting test for all the odd electrodes concurrently (large droplets are now on even electrodes).

4. Route the droplets consecutively to a capacitive sensing circuit for test readouts.

5. Repeat the test procedure for the next row.

6. Repeat steps 1-5 for columns.

Note that in the above method, only one capacitive sensing circuit is used, therefore the hardware cost is significantly reduced. However, additional droplet routing steps are needed. In order to minimize the number of droplet manipulations, test results are read out not directly after each splitting or merging test, but after the both of them are carried out, as shown in the above steps. As a trade-off, a more complicated test result interpretation scheme is required.

If all the tests in one row are executed without the detection of a malfunction, droplet volume should be almost the same. However, if a malfunction occurs, volume variation is expected, as shown in Fig. 11.

In Fig.11, the shaded droplet undergoes a unbalanced split during the splitting test. Since all other droplets are split evenly, this malfunction results in a pair of test droplets of abnormal volume, one bigger and the other smaller. If the next step of test yields no malfunction, the droplet volume variation is propagated one electrode away. Therefore, we can easily detect the malfunction by checking the test results.

The proposed test method achieves high efficiency. An implicit assumption here is that adjacent electrodes are not defective. Such defects can be detected by separate structural test [21]. For an $N \times N$ array, only $N^{2}+N$ manipulation steps are needed, while the test method in prior work [21] requires $4 N^{2}$ steps. Moreover, the method uses only one capacitive sensing circuit, irrespective of the array size. This is in contrast to [21], which requires $N^{2}$ capacitive sensing circuits for an $N \times N$ microfluidic array. 
The reduction in production cost is therefore significant.

\section{Experimental and Simulation Results}

In this section, we apply the proposed functional test methods to a fabricated chip. The chip-under-test is a PCB microfluidic platform for the Polymerase Chain Reaction (PCR), as shown in Fig. 12. The platform consists of two columns and two rows of electrodes, three reservoirs, and routing electrodes that connect the reservoirs to the array.

Next we applied the proposed functional test methods to the chip. Dispensing test and routing test are trivial due to the simple structure of the chip. Therefore, we only focused on the mixing and splitting tests. Following the steps in Section 4.3, we first targeted the bottom row and dispensed five test droplets to the odd electrodes, as shown in Fig. 13(a).

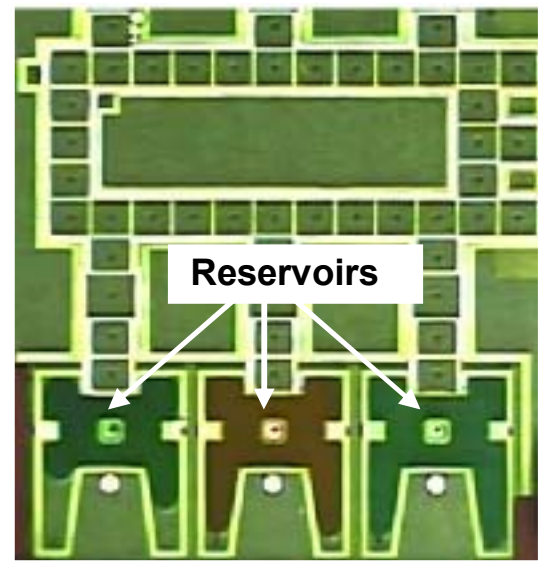

Fig. 12: Fabricated biochip for PCR.

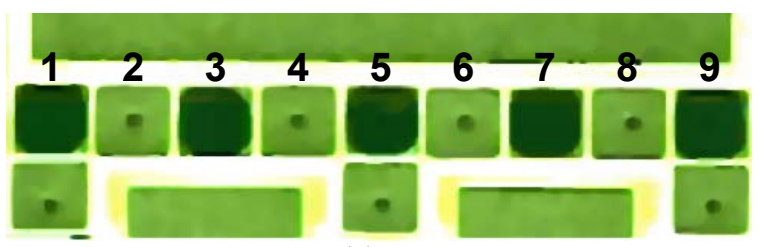

(a)

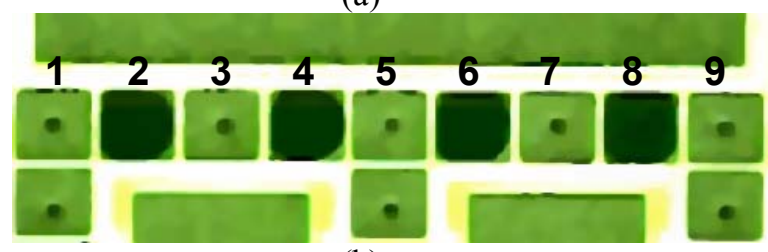

(b)

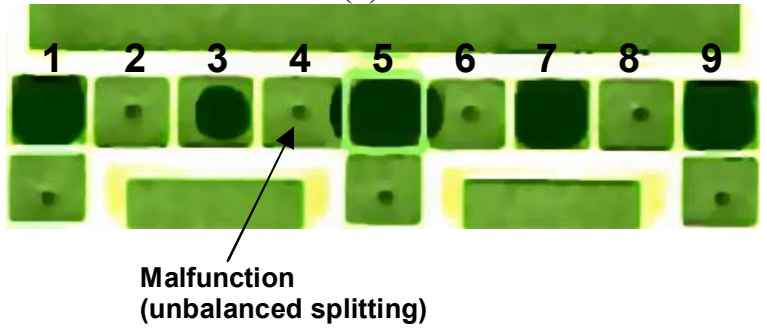

(c)

Fig. 13: Mixing and splitting test for a fabricated PCR chip (bottom row of Fig. 12).

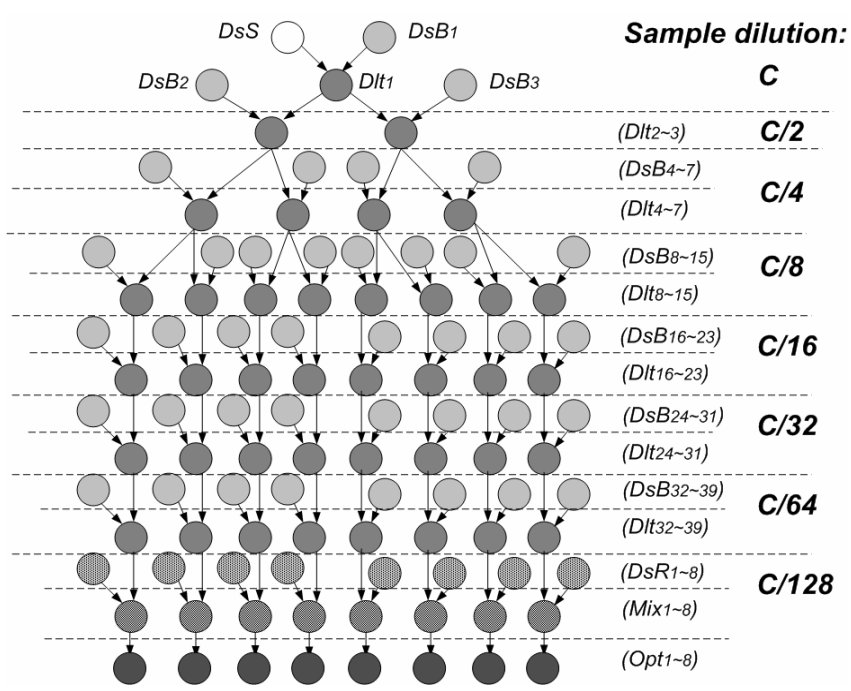

Fig. 14: Sequencing graph for a protein assay.

Table 2: Experimentally-characterized module library for synthesis.

\begin{tabular}{|l|l|c|}
\hline Operation & Resource & Time (s) \\
\hline DsS;DsB; DsR & On-chip reservoir/dispensing port & 7 \\
\hline \multirow{4}{*}{ Dlt } & $2 \times 2$-array dilutor & 12 \\
\cline { 2 - 3 } & $2 \times 3$-array dilutor & 8 \\
\cline { 2 - 3 } & 2x4-array dilutor & 5 \\
\cline { 2 - 3 } & 4-electrode linear array dilutor & 7 \\
\hline \multirow{4}{*}{ Mix } & 2x2-array mixer & 10 \\
\cline { 2 - 3 } & 2x3-array mixer & 6 \\
\cline { 2 - 3 } & 2x4-array mixer & 3 \\
\cline { 2 - 3 } & 4-electrode linear array mixer & 5 \\
\hline Opt & LED+Photodiode & 30 \\
\hline Storage & Single cell & N/A \\
\hline
\end{tabular}

Then splitting test of the even electrodes was carried out. Droplets were split and merged on the even electrodes. In Fig. 13(b), we see a series of droplets of the same volume resting on the even electrodes, which means that all the odd electrodes passed the splitting test, and merging at the even electrodes worked well. However, when we carried out the splitting test on the even electrodes, a large variation in droplet volume was observed on the $3^{\text {rd }}$ and $5^{\text {th }}$ electrodes; see Fig. 13(c). This variation implied a malfunction, leading to unbalanced splitting on the $4^{\text {th }}$ electrode. The malfunction was detected when the droplets were routed to the capacitive sensing circuit. We then labeled the $4^{\text {th }}$ electrode on the bottom row as an unqualified splitting site so that synthesis tools will not map a splitter to it. Thus the system robustness of the synthesized design was enhanced.

We next evaluate the improvement in system robustness using a biochip for a real-life protein assay [22]. Based on the Bradford reaction [22], the protocol for a generic droplet-based colorimetric protein assay is as follows. First, a droplet of the sample, such as serum or some other physiological fluid containing protein, is generated and dispensed into the biochip. Buffer droplets, such as $1 \mathrm{M}$ $\mathrm{NaOH}$ solution, are then introduced to dilute the sample to obtain a desired dilution factor $(D F)$. This on-chip dilution 
is performed using multiple hierarchies of binary mixing/splitting phases, referred to as the interpolating serial dilution method [1]. The mixing of a sample droplet of protein concentration $C$ and a unit buffer droplet results in a droplet with twice the unit volume, and concentration $C / 2$. Splitting this large droplet results in two unit-volume droplets of concentration $C / 2$ each. Continuing this step in a recursive manner using diluted droplets as samples, an exponential dilution factor of $D F=2^{N}$ can be obtained in $N$ steps. After dilution, droplets of reagents, such as Coomassie brilliant blue G-250 dye, are dispensed into the chip, and they mix with the diluted sample droplets. Next the mixed droplet is transported to a transparent electrode, where an optical detector (e.g., a LED-photodiode setup) is integrated. The protein concentration can be measured from the absorbance of the products of this colorimetric reaction using a rate kinetic method [20]. Finally, after the assay is completed, all droplets are transported from the array to the waste reservoir. A sequencing graph model can be developed from the above protocol for a protein assay $(D F$ $=128$ ), as shown in Figure 5. There are a total of 103 nodes in one-to-one correspondence with the set of operations in a protein assay, where DsS, $\operatorname{DsB}_{i}(i=1, \ldots, 39)$, and $\operatorname{DsR}_{i}(i=$ $1, \ldots, 8)$ represents the generation and dispensing of sample, buffer and reagent droplets, respectively.

In addition, $\operatorname{Dlt}_{i}(i=1, \ldots, 39)$ denotes the binary dilution (including mixing/splitting) operations, $\operatorname{Mix}_{i}(i=1, \ldots, 8)$ represents the mixing of diluted sample droplets, and reagent droplets; $\mathrm{Opt}_{i}(\mathrm{i}=1, \ldots, 8)$ denotes the optical detection of the droplets. Until the fourth step of a serial dilution, all diluted sample droplets are retained in the microfluidic array. After that stage, for each binary dilution step, only one diluted sample droplet is retained after splitting, while the other droplet is moved to the waste reservoir.

The basic operations for protein assay have been implemented on a digital microfluidic biochip [1, 22]. Experiments indicate that the dispensing operation takes 7 seconds [1]. The operation times of various mixers have been found to be different [1]. A binary dilution operation can also be easily implemented by mixing of sample droplet followed by droplet splitting. Absorbance of the assay product can be measured using an integrated LED-photodiode setup. Experiments indicate this absorbance measurement takes 30 seconds [22]. The microfluidic module library for a protein assay is shown in Table 2. A $10 \times 10$ microfluidic array is used to execute the assay.

Next we evaluate system dependability of the synthesized design for three cases: (i) no testing is carried out; (ii) only structural test is carried out; (iii) functional test is carried out. A design is deemed to fail if any module suffers from a defect or a malfunction, e.g., a mixer suffering from a faulty mixing site.

We next generate 200 simulated samples of faulty arrays. Each faulty array is derived by randomly injecting fault (due to defects and malfunctions) in the array. Note that we do not specify the types of injected malfunctions.
Here we simply assume that all the injected malfunctions can be detected by the proposed functional test and cannot be detected through structural test. Let A be the event that a unit cell has a defect. We let the defect probability $p=\mathrm{P}[\mathrm{A}]$ take two values, namely 0.01 and 0.05 . Let $\mathrm{B}$ be the event that a group of electrodes suffer from a malfunction. Since a defective unit cell leads to a malfunction of the module where it is used, we are interested here in the conditional probability $q=\mathrm{P}[\mathrm{B} \mid \overline{\mathrm{A}}]$, i.e., the probability that a module is malfunctioning even if there is no defect in it. We consider four values of $q$, namely $0.02,0.04,0.06,0.08$. For each faulty array, the structural test method from [7] is carried out to detect and locate defect sites.

Next the synthesis method from [8] is used to map the protein array on to defect-free regions of the array. We also use functional test to detect and locate malfunctions in the array. These malfunctions are then bypassed during the synthesis of the chip for the protein array. As a baseline, we also carry out the synthesis for an array to which neither structural test nor functional test have been applied.

First we determine the failure rate $R, 0 \leq R \leq 1$, for the three scenarios when the protein assay is mapped to an array with defects and malfunctions. When functional testing is carried out, the failure rate due to target defects and malfunctions is zero because all of them are detected by the test procedure. If no testing is carried out, the failure rate is as high as 0.85 , i.e., the protein assay fails for as much as $85 \%$ of the 200 simulated chips. If structural testing is used, the failure rate is lower, but it is still significant —as high as 0.75 .

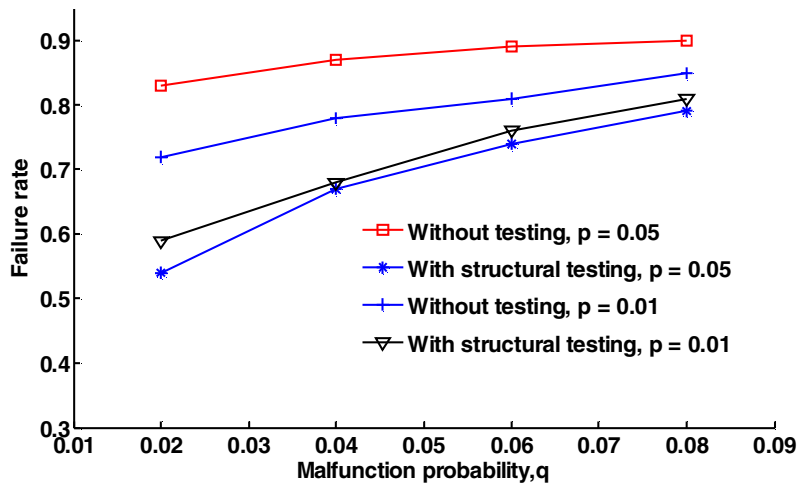

Fig. 15: Failure rate for synthesized design without testing and with structural testing.

Fig. 15 shows that as the malfunction probability increases, the failure rate $R$ becomes considerable even when structural testing is used. Moreover, the benefits of structural testing are less evident for the smaller value of the defect probability, i.e., $p=0.01$. Therefore, functional testing is needed to augment droplet-transportation-based structural testing for digital microfluidic arrays. A counter-intuitive finding from Fig. 15 is that the failure rate is lower for $p=0.05$ compared to $p=0.01$. This occurs because large $p$ implies that there is low likelihood of a defect-free cell. Hence structural testing is likely to catch such defects. 


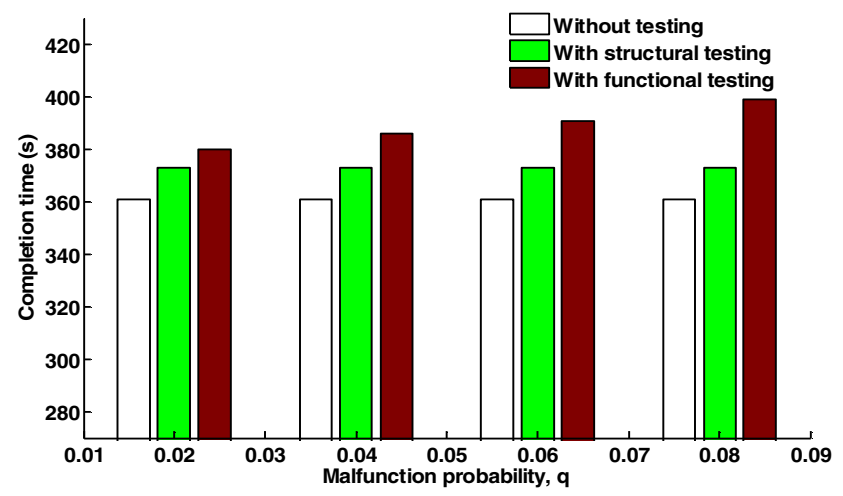

(a)

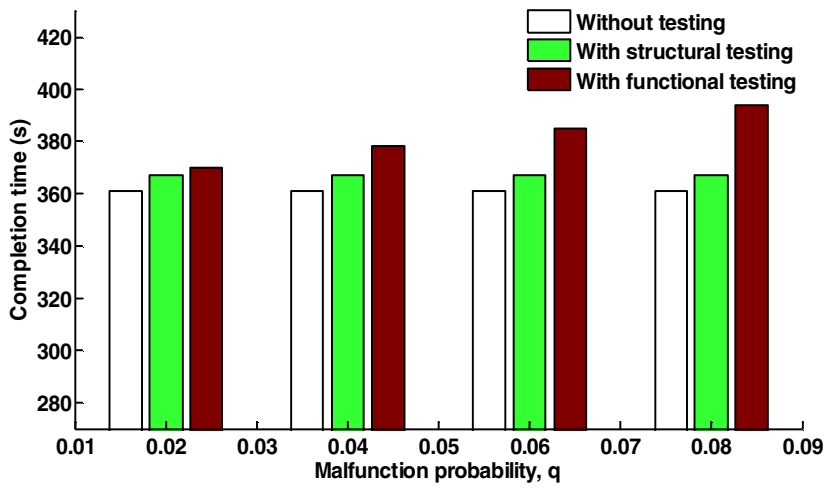

(b)

Fig. 16: Bioassay completion time for synthesized design without test, with structural test, and with functional test for defect occurrence probability of (a) $\mathrm{P}[\mathrm{A}]=\mathbf{0 . 0 1}$. (b) $\mathrm{P}[\mathrm{A}]=\mathbf{0 . 0 5}$.

The detection of more defects and malfunctions using functional testing leads to a corresponding increase in the assay completion time. This happens because fault detection and fault location leave fewer unit cells available for the protein assay, and the synthesis procedure employs less parallelism in the execution of the microfluidic operation. Fig. 16 shows the assay completion time for the three scenarios that we are considering for the protein assay. As expected, the bioassay time is slightly higher when functional testing is used, and the increase is more for higher values of the malfunction probability $q$. This increase is acceptable because functional testing ensures that the assay will run to completion if the fluidic operations are mapped only on qualified region of the array.

\section{Conclusions}

We have presented several techniques for the functional testing of droplet-based microfluidic biochips. These techniques address fundamental biochip operations such as droplet dispensing, droplet transportation, mixing, splitting, and capacitive sensing. Functional testing is carried out using parallel droplet pathways, and it leads to qualified regions where synthesis tools can map microfluidic functional modules. We have demonstrated functional testing for a fabricated biochip used for PCR. We have also presented simulation results for a protein assay, and quantified the small increase in assay completion time that is needed to achieve $100 \%$ coverage of the target defects and malfunctions with functional testing.

\section{References}

[1] R. B. Fair, V. Srinivasan, H. Ren, P. Paik, V. K. Pamula and M. G. Pollack, "Electrowetting-based on-chip sample processing for integrated microfluidics," Proc. IEEE International Electron Devices Meeting (IEDM), 2003, pp. 32.5.1-32.5.4.

[2] E. Verpoorte and N. F. De Rooij, "Microfluidics meets MEMS”, Proc. IEEE, 2003, vol. 91, pp. 930-953.

[3] J. Zeng and T. Korsmeyer, "Principles of droplet electrohydrodynamics for lab-on-a-chip", Lab on a Chip, 2004, vol. 4, pp. 265-277.

[4] R. B. M. Schasfoort, S. Schlautmann, J. Hendrikse and A. van den Berg, "Field-effect flow control for microfabricated fluidic networks", Science, 1999, vol. 286, pp, 942-945.

[5] B. M. Paegel, R. G. Blazej and R. A. Mathies, "Microfluidic devices for DNA sequencing: sample preparation and electrophoretic analysis", Current Opinion Biotechnol., 2003, vol.14, pp. 42-50.

[6] J. Gong, and C. J. Kim, "Two-dimensional digital microfluidic system by multi-layer printed circuit board", Proc. IEEE MEMS Conference, 2005, pp. 726-729.

[7] F. Su, S. Ozev and K. Chakrabarty, "Testing of droplet-based microelectrofluidic systems", Proc. International Test Conference, 2003, pp. 1192-1200.

[8] F. Su and K. Chakrabarty, "Unified high-level synthesis and module placement for defect-tolerant microfluidic biochips", Proc. IEEE/ACM Design Automation Conference, 2005, pp. 825-830.

[9] N. Deb and R. D. Blanton, "Analysis of failure sources in surface-micromachined MEMS", Proc. International Test Conference, 2000, pp. 739-749.

[10] A. Dhayni, S. Mir, L. Rufer and A. Bounceur, "Pseudorandom functional BIST for linear and nonlinear MEMS", Proc. Design Automation and Test in Europe (DATE), 2006, pp. 664-669.

[11] H. G. Kerkhoff, "Testing of microelectronic-biofluidic systems", IEEE D\&T for Computers, 2007, vol. 24, pp. 72-82.

[12] H. G. Kerkhoff and M. Acar, "Testable design and testing of micro-electro-fluidic arrays", Proc. IEEE VLSI Test Symposium, 2003, pp. 403-409.

[13] F. Su, S. Ozev and K. Chakrabarty, "Ensuring the operational health of droplet-based microelectrofluidic biosensor systems", IEEE Sensors, 2005, vol. 5, pp. 763-773.

[14] F. Su, S. Ozev and K. Chakrabarty, "Concurrent testing of droplet-based microfluidic systems for multiplexed biomedical assays", Proc. International. Test Conference, 2004, pp. 883-892.

[15] T. H. Schulte, R. L. Bardell and B. H. Weigl, "Microfluidic technologies in clinical diagnostics", Clinica Chimica Acta, 2002, vol. 321, pp. 1-10. 
[16] F. Su and K. Chakrabarty, "Defect-oriented testing and diagnosis of digital microfluidics-based biochips", Proc. IEEE International Test Conference, 2005, pp. 487-496.

[17] V. Hlady, R. A. Wagenen and J. D. Andrade, Surface and Interfacial Aspects of Biomedical Polymers: Protein Adsorption; Andrade, J. D. Ed.; Plenum Press: New York, 1985, 2, pp. 81.

[18] M. G. Pollack, P. Y. Paik, A. D. Shenderov, V. K. Pamula, F. S. Dietrich and R. B. Fair, "Investigation of electrowetting-based microfluidics for real-time PCR applications", International Conference on Micro Total Analysis Systems (uTAS), 2003.

[19] P. Paik, V. K. Pamula, M. G. Pollack and R. B. Fair, "Rapid droplet mixers for digital microfluidic systems", Lab on a Chip, 2003, vol. 3, pp. 253-259.

[20] P. Paik, V. K. Pamula, M. G. Pollack and R. B. Fair, "Electrowetting-based droplet mixers for microfluidic systems", Lab on a Chip, vol. 3, 2003, pp. 28-33.

[21] F. Su, W. Hwang, A. Mukherjee and K. Chakrabarty, "Testing and diagnosis of realistic defects in digital microfluidic biochips", Journal of Electronic Testing: Theory and Applications, 2007, vol. 23, pp. 219-233.

[22] V. Srinivasan, V. K. Pamula, P. Paik, and R. B. Fair, "Protein stamping for MALDI mass spectrometry using an electrowetting-based microfluidic platform", Proc. SPIE, 2004, vol. 5591, pp. 26-32. 